of gastrointestinal mucosal barrier. II. The effect of natural versus artificial feeding on intestinal permeability to macromolecules. Pediatr Res 15:245249

26. Stern M, Pang KY, Walker WA 1984 Food proteins and gut mucosal barrier. II. Differential interaction of cow's milk proteins with the mucous coat and the surface membrane of adult and immature rat jejunum. Pediatr Res $18: 1252-1257$
27. Dave JR, Knazek RA, Liu SC 1981 Prolactin modifies the fluidity of rat liver membranes. Biochem Biophys Res Commun 100:45-51

28. Allen A, Bell A, Mantle M, Pearson JP 1982 The structure and physiology of gastrointestinal mucus. Adv Exp Biol Med 144:115-133

29. Lamm ME, Weisz-Carrington P, Roux ME, McWilliams M, Phillips-Quagliata JM 1978 Development of the IgA system in the mammary gland. Adv Exp Biol Med 107:35-42

\title{
Effect of Maternal Oxygen Inhalation on the Fetus with Growth Retardation
}

\author{
RITA A. VILEISIS \\ Department of Pediatrics, Duke University Medical Center, Durham, North Carolina 27710
}

\begin{abstract}
Decreased nutrient and oxygen transfer to the fetus accounts for fetal growth retardation in pregnancies complicated by severe uteroplacental insufficiency. A model of uteroplacental insufficiency was produced by ligation of the uterine artery of one horn in pregnant rats at 17 days of gestation. The pregnant rats were assigned to environmental chambers containing a gas mixture of either an increased fractional inspired oxygen concentration of $0.40\left(\mathrm{O}_{2}\right)$ or room air from day 17 through 21 of gestation. Supplemental oxygen inhalation resulted in increased survival of the fetuses from the ligated horn [34 \pm $6 \%$ in intrauterine growth retardation (IUGR)-room air versus $57 \pm 8 \%$ in IUGR-O weight, expressed as a percentage of nonligated appropriate for gestational age control littermates $(67 \pm 2 \%$ in IUGR-room air versus $74 \pm 2 \%$ in IUGR-O $\mathrm{O}_{2}$ ). A role for hypoxia in the suppression of fatty acid synthesis in IUGR fetal tissues had been postulated; however, maternal oxygen inhalation did not result in any increase in fatty acid content or specific activity in liver, lung, or carcass of IUGR fetuses. These data indicate that supplemental maternal oxygen inhalation improves survival and growth of fetuses compromised by uteroplacental insufficiency, but it has no apparent effect on lipogenesis at term. (Pediatr Res 19: 324-327, 1985)
\end{abstract}

\section{Abbreviations}

IUGR, intrauterine growth retardation

RA, room air

AGA, appropriate for gestational age ip, intraperitoneal

Fetal growth is dependent on placental function, maternal nutrition, as well as many other factors. Limitation of blood flow

Received August 17, 1984; accepted November 13, 1984.

Address reprint request to R. A. Vileisis, M.D.. Duke University Medical Center, Box 3967, Durham, NC 27710.

Supported in part by the Perry Como Children's Fund, Duke University Medical Center.

This work was presented in part at the 25th Annual Meeting of the American College of Nutrition, Boston, MA, September, 1984. to the pregnant uterus restricts availability of all potential metabolic substrates to the placenta both for its own metabolic needs and for subsequent delivery to the growing fetus. Placental transfer of analogs of glucose and amino acids correlates directly with maternal placental blood flow in fetuses with spontaneous and experimentally induced growth retardation $(13,17)$. In addition, low uteroplacental blood flow results in decreased oxygen delivery to the placenta $(4,11,14)$.

Hypoxemia impairs the placenta's capacity to transfer glucose (9) and amino acids (8), thus contributing an additional mechanism for fetal growth retardation in pregnancies complicated by uteroplacental insufficiency. Furthermore, severe restriction of uterine blood flow has been shown in several animal models to result in fetal hypoxemia, and thus, contribute to growth retardation (12). The causal relationship between hypoxia and IUGR may be mediated by decreased release of growth promoting factors, and/or decreased synthetic capability due to decreased fetal oxygen consumption.

The purpose of this study was to determine the effect of increased ambient oxygen concentration in the maternal environment on the IUGR fetus. The animal model chosen was one in which all potential substrates for fetal growth were severely limited by means of uterine artery ligation, and then only one factor in the maternal environment was augmented, oxygen. Fatty acid synthesis was evaluated because it is a synthetic function that is known to be impaired in the IUGR conceptus (19), is responsive to improved nutrient availability (20), and is dependent on adequate oxygen supply (21).

\section{MATERIALS AND METHODS}

Twenty-eight pregnant Sprague-Dawley rats weighing between 250 and $325 \mathrm{~g}$ were obtained from the Charles River Laboratories (Wilmington, MA) and allowed free access to a stock diet. Rats had been mated between 19.00 and $11.00 \mathrm{~h}$. The next morning was considered to be day 0 of pregnancy. On day 17 of gestation, under ketamine anesthesia (20-30 mg, ip), a laparotomy incision was made. The uterus was exposed and a 3-0 silk ligature placed around the uterine artery supplying the uterine horn with the larger number of fetuses to produce uteroplacental insufficiency as described by Wigglesworth (23). The vasculature of the opposite uterine horn and ovarian artery of the ligated horn were left untouched. The fetuses from the nonligated horn served as 
the appropriately grown controls (AGA). The number of fetuses in each horn was recorded. The uterus was returned to the abdominal cavity and the incision was closed. The pregnancy was allowed to continue until day 21 of gestation.

After surgery, all animals were kept in individual cages, in the same room with a uniform light/dark cycle. Eleven animals were assigned to the increased fractional ambient oxygen concentration environment $\left(\mathrm{O}_{2}\right)$ of $0.40 \pm 0.02$ provided by a mixture of compressed oxygen and air. The oxygen concentration was monitored twice daily with an oxygen analyzer and minimal adjustments were made if necessary. The control animals were housed in identical cages except compressed air (RA) was provided into the environmental chamber $(n=17)$. Fractional concentration of oxygen was verified to be 0.21 in these chambers. During the 4-day period of time, the animals were unrestrained, allowed free access to stock diet and water, and kept in their respective chambers without being disturbed until day 21 of gestation.

On day 21 , animals received $1 \mathrm{mCi}^{3} \mathrm{H}_{2} \mathrm{O}$ ip, and food and water were withdrawn. Four hours later the mother was sacrificed, serum collected, and the fetuses were quickly delivered by Cesarean section. Resorbed fetuses and placentas were discarded. Fetal blood was collected via a deep neck incision. After exsanguination, the fetuses were weighed individually and fetal organs were dissected. Organs from all viable fetuses from the same horn were pooled for analysis of fatty acid synthesis.

Lipid from pooled fetal organs was extracted by the BlighDyer method (2). After saponification with ethanolic $\mathrm{KOH}$, the nonsaponifable lipids were extracted with hexane and discarded. The hydrolysate was acidified and fatty acids extracted with hexane. Aliquots of the extract were used for determination of radioactivity and for titration to determine the fatty acid content. An automated titration system (Radiometer) dispensed $0.01 \mathrm{~N}$ $\mathrm{NaOH}$; Nile blue was used as the indicator dye. One mM palmitic acid was used as a standard. Fatty acid content is expressed as $\mathrm{cpm} / \mu \mathrm{mol}$ of fatty acid.

Statistical analysis was performed by the paired $t$ test between fetuses from the ligated uterine horn and littermates from the nonmanipulated uterine horn of the same pregnant rat. Significance is assigned to a $p<0.05$. If there was a significant difference between the AGA and IUGR fetuses of the RA group, the ratio of IUGR:AGA was calculated to eliminate interlitter variability and to permit comparison of the RA and $\mathrm{O}_{2}$ groups by unpaired $t$ test.

To estimate the effect of supplemental oxygen on the maternal arterial tension, eight additional animals were studied on day 20 of gestation under light ketamine (15 mg ip) anesthesia. While restrained on their backs, pregnant rats were administered either room air $(n=4)$ or $\mathrm{FiO}_{2}$ of $0.40(n=4)$ via a head hood placed over the head and upper thorax. Blood was obtained from the maternal abdominal aorta or ovarian artery and the blood gases were determined on a Radiometer ABL-2.

\section{RESULTS}

Maternal weight gain (mother plus products of conception) was similar in both groups. Over the 4-day period the room air control group gained $14.9 \pm 2.7 \mathrm{~g}(\overline{\mathrm{x}} \pm \mathrm{sem} ; n=17)$ as compared to $19.3 \pm 3.0 \mathrm{~g}$ in the oxygen supplemented group $(n=11)$. Pregnant rats in RA had a mean arterial pH of $7.46 \pm 0.05, \mathrm{PCO}_{2}$ of $36 \pm 4$ torr, and $\mathrm{PO}_{2}$ of $67 \pm 5$ torr, as compared to a mean arterial pH of $7.38 \pm 0.03, \mathrm{PCO}_{2}$ of $42 \pm 3$ torr, and $\mathrm{PO}_{2}$ of 185 \pm 38 torr $\left(p<0.05\right.$ for $\mathrm{O}_{2}$ values) in the pregnant rats exposed to a fractional oxygen concentration of 0.40 .

At day 17 of gestation, the number of implantation sites were similar in both ligated horns $(7.3 \pm 0.4$ in IUGR-RA versus 7.5 \pm 0.6 in IUGR- $\mathrm{O}_{2}$ ) and nonligated horns $(4.1 \pm 0.2$ in AGA-RA versus $4.7 \pm 0.4$ in $\mathrm{AGA}-\mathrm{O}_{2}$ ). Fetuses from the nonligated horns of both groups of mothers had similar survival $(98 \%$ in RA versus $94 \%$ in $\mathrm{O}_{2}$ ), but fetuses from the ligated horns had increased survival if the mothers had oxygen supplementation (57 $\pm 8 \%$ IUGR-O ${ }_{2}$ versus $34 \pm 6 \%$ IUGR-RA). Five of the 17
RA litters had no viable fetuses from the ligated horn (data excluded in further analysis); all $\mathrm{O}_{2}$ litters had two or more viable growth-retarded fetuses.

Uterine artery ligation resulted in significant growth impairment in both groups. Weight of fetuses from nonligated horns was unchanged by oxygen supplementation $(4.61 \pm 0.19 \mathrm{~g}$ in AGA-RA versus $4.58 \pm 0.14 \mathrm{~g}$ in $\mathrm{AGA}-\mathrm{O}_{2}$ ), while the weight of fetuses from ligated horns of mothers from the oxygen enriched environment was increased $(3.11 \pm 0.20$ in IUGR-RA versus $3.42 \pm 0.14$ in IUGR-O ${ }_{2}$ ). This increase in weight is significant when the interlitter variability is eliminated by expressing the weight of the fetuses from the ligated horn as a percentage of their own nonmanipulated littermates: the IUGR-RA fetuses weighed $67 \pm 2 \%$ (range $53-76 \%$ ) of their AGA littermates, while IUGR-O $\mathrm{O}_{2}$ fetuses weighed $74 \pm 2 \%$ (range $63-81 \%$ ) of their nonligated counterparts.

In the room air group, two litters had a single fetus from the ligated horn whose weight was greater than its lightest counterpart from the nonligated horn. In comparison, the oxygen supplemented group had five of 11 litters in which the weight of at least one fetus from the ligated horn exceeded its lightest nonligated counterpart.

Individual organ weights confirmed previously demonstrated decreases in brain, liver, lung, and carcass tissue from growthretarded fetuses $(19,23)$. Oxygen supplementation to the maternal rat did not result in significant increases in fetal individual organ weights (Table 1), although most organs were slightly heavier.

With the exception of the brain, fatty acid content (Table 2) was decreased in all organs of IUGR pups which were studied. Maternal oxygen supplementation during the time of uteroplacental insufficiency did not alter fatty acid content. Fatty acid synthetic rates of liver, lung, and carcass differed between AGA and IUGR fetuses of mothers exposed to room air (Table 3). Specific activity measurements of ${ }^{3} \mathrm{H}$-fatty acid in brain and placenta do not demonstrate differences between IUGR and AGA fetuses in either the room air or oxygen group. Oxygen

Table 1. Fetal morphometric data $(\bar{x} \pm S E M)$

\begin{tabular}{lccccc}
\hline & \multicolumn{2}{c}{ RA } & & \multicolumn{2}{c}{ Oxygen } \\
\cline { 2 - 3 } \cline { 5 - 6 } \cline { 5 - 6 } & AGA & IUGR & & AGA & IUGR \\
\hline Survival* $^{n}$ & $97 \pm 3$ & $34 \pm 6$ & & $94 \pm 4$ & $57 \pm 8 \dagger$ \\
Fetal wt (g) & $4.61 \pm 0.19$ & $3.11 \pm 0.20 \ddagger$ & $4.58 \pm 0.14$ & $3.42 \pm 0.14 \ddagger$ \\
Fetal wt§ & & $67 \pm 2$ & & $74 \pm 2 \ddagger$ \\
Brain (mg) & $183 \pm 6$ & $161 \pm 8 \ddagger$ & & $185 \pm 5$ & $169 \pm 4 \ddagger$ \\
Liver (mg) & $348 \pm 17$ & $182 \pm 19 \ddagger$ & & $349 \pm 10$ & $219 \pm 9 \ddagger$ \\
Lung (mg) & $135 \pm 17$ & $91 \pm 17 \ddagger$ & & $110 \pm 3$ & $83 \pm 6 \ddagger$ \\
Placenta (mg) & $500 \pm 23$ & $413 \pm 27 \ddagger$ & & $506 \pm 19$ & $440 \pm 15 \ddagger$ \\
Carcass (g) & $3.2 \pm 0.1$ & $2.2 \pm 0.1 \ddagger$ & $3.2 \pm 0.1$ & $2.4 \pm 0.1 \ddagger$ \\
\hline
\end{tabular}

* Percentage viable fetuses at day 21 of those present at day 17 gestation.

$\dagger p<0.05$ IUGR- $\mathrm{O}_{2}$ fetuses compared to IUGR-RA.

$\ddagger p<0.05$ IUGR compared to own AGA littermate of same gas mixture inhalation group.

$\S$ IUGR weight expressed as percentage of AGA littermate weight.

Table 2. Fatty acid content ( $\mu$ mol/organ, $\bar{x} \pm S E M$ )

\begin{tabular}{lrcrrr}
\hline & \multicolumn{2}{c}{ RA } & & \multicolumn{2}{c}{ Oxygen } \\
\cline { 2 - 3 } \cline { 5 - 6 } & \multicolumn{1}{c}{ AGA } & IUGR & \multicolumn{1}{c}{ AGA } & \multicolumn{1}{c}{ IUGR } \\
\hline Brain & $9.5 \pm 0.3$ & $9.0 \pm 0.3$ & & $9.2 \pm 0.4$ & $8.9 \pm 0.3$ \\
Liver & $13.4 \pm 0.3$ & $9.1 \pm 0.4^{*}$ & & $13.1 \pm 0.5$ & $9.6 \pm 0.4^{*}$ \\
Lung & $6.7 \pm 0.4$ & $4.1 \pm 0.6^{*}$ & $6.6 \pm 0.4$ & $4.5 \pm 0.3^{*}$ \\
Placenta & $15.9 \pm 0.9$ & $13.1 \pm 0.6^{*}$ & & $15.5 \pm 0.9$ & $13.1 \pm 0.6^{*}$ \\
Carcass & $70.4 \pm 2.4$ & $51.4 \pm 3.0^{*}$ & & $71.1 \pm 2.1$ & $56.7 \pm 2.2^{*}$ \\
\hline
\end{tabular}

${ }^{*} p<0.05$ IUGR compared to AGA of same gas mixture inhalation group. 
Table 3. Specific activity $\left({ }^{\beta} H \mathrm{cpm} / \mu M\right.$ fatty acid, $\left.\bar{x} \pm S E M\right)$

\begin{tabular}{|c|c|c|c|c|c|c|}
\hline & \multicolumn{3}{|c|}{ RA } & \multicolumn{3}{|c|}{ Oxygen } \\
\hline Brain & $39.9 \pm 2.3$ & $41.5 \pm 5.3$ & & $37.3 \pm 1.4$ & $36.2 \pm 1.6$ & \\
\hline Lung & $56.8 \pm 4.7$ & $42.5 \pm 3.5^{*}$ & $76 \pm 4$ & $47.3 \pm 4.0$ & $39.0 \pm 2.0$ & $83 \pm 7$ \\
\hline Placenta & $9.5 \pm 0.8$ & $8.5 \pm 0.8$ & & $9.2 \pm 2.0$ & $7.0 \pm 0.7$ & \\
\hline Carcass & $39.4 \pm 2.8$ & $35.5 \pm 2.6^{*}$ & $91 \pm 5$ & $36.3 \pm 1.7$ & $31.6 \pm 1.5^{*}$ & $88 \pm 5$ \\
\hline
\end{tabular}

${ }^{*} p<0.05$ comparing IUGR with AGA of same gas mixture inhalation group.

Comparison of data from each litter expressed as ratio IUGR/AGA shows no statistical differences between RA and $\mathrm{O}_{2}$ groups.

supplementation did not change fatty acid synthesis rate in the liver or carcass from the AGA group nor did it improve the decreased fatty acid synthesis rate of the IUGR fetal organs. Rates of fatty acid synthesis in the lungs of the IUGR- $\mathrm{O}_{2}$ fetuses more closely approximated those of their AGA counterparts than did the IUGR-RA lungs. The lack of difference in lung fatty acid specific activity in the $\mathrm{O}_{2}$ group is due to an apparent decrease in the rate of synthesis in the $\mathrm{AGA}-\mathrm{O}_{2}$ lung rather than an increase in fatty acid synthesis rate in the IUGR-O $\mathrm{O}_{2}$ tissue. This decrease is probably physiologically insignificant since the rates of fatty acid synthesis of $A G A-R A$ lung and $A G A-O_{2}$ lung were equivalent $(p=0.15)$.

\section{DISCUSSION}

Although the classic model of severe chronic reduction in uteroplacental blood flow has been extensively studied, the relative contribution of nutrient versus oxygen deprivation in causing the fetal growth retardation associated with uteroplacental insufficiency has not been elucidated. In this study design, uterine artery ligation restricted the delivery of all potential fetal substrates and only one alterable factor in the maternal environment, that of oxygen, was augmented to determine the effect of such manipulation on the IUGR conceptus.

In this study the supplemental maternal oxygen inhalation overcame, in part, the growth retardation caused by restricted uterine blood flow. Presumably the increase in placental oxygen transfer and subsequent increase in fetal oxygen tension accounts for the improved growth and for the increase in fetal survival.

Fetal uptake of substrates and subsequent growth are determined by maternal substrate concentration, blood flow to the placenta, and transport mechanisms in the placenta. In several animal species a direct correlation has been demonstrated between maternal placental blood flow and fetal weight $(13,17)$, suggesting that maternal placental blood flow determines substrate transfer.

Oxygen delivery to the fetus is dependent on both maternal placental blood flow and maternal blood concentration of that substrate (11). Under normal conditions the oxygen supply to the fetus is approximately twice that which the fetus needs for adequate oxygen uptake and normal acid base balance (24). Significant reduction of uterine blood flow will result in decreased fetal oxygen uptake. With severe uterine hypoperfusion, i.e. less than $50 \%$ of the uterine blood flow, the placenta does not improve in its efficiency for gaseous exchange, and fetal oxygen uptake becomes dependent on the rate of oxygen delivery to the pregnant uterus. Consequently, fetal hypoxemia and growth retardation ensue. Localized placental hypoperfusion, as caused by injections of microspheres (3) into the uteroplacental vascular bed or by removal of endometrial caruncles (16), also leads to reduction in fetal arterial oxygen tension and growth impairment. Pure hypoxemia has been shown to limit the growth of the chick embryo (10). Other models of fetal hypoxemia without blood flow or nutrient limitation, such as maternal arterial desaturation $(5,6,18)$, also cause growth retardation.

The potential mechanism(s) by which maternal hyperoxia improves fetal survival and growth are not clear from this study. One possibility is hyperoxia causes a redistribution of blood flow in favor of the fetus. However, the Greiss et al. (7) data show no effect of hyperoxia on uterine blood flow. By itself, however, increased maternal arterial $\mathrm{PO}_{2}$ of a magnitude similar to that observed in this study would be expected to result in an increase in placental oxygen transfer and in umbilical venous $\mathrm{PO}_{2}(15)$. Alternately, correction of placental hypoxemia could restore to normal glucose and amino acid transfer to the fetus $(8,9)$, if substrate deficiency, rather than hypoxemia, were the direct cause of growth retardation. An additional explanation for improved fetal growth is that supplemental maternal oxygen inhalation resulted in a change in the fetal hormonal milieu. Given that hypoxia inhibits insulin release (1) and that insulin may be the intrauterine growth hormone, an increased circulating insulin concentration might account for greater fetal weight. Finally, it is possible that the hyperoxic mother has a greater food intake, thus providing her fetuses with supplemental nutrients. The lack of difference in weight gain between the hyperoxic and normoxic groups, however, is not consistent with this hypothesis.

Rates of lipogenesis of the IUGR fetus can be increased to approximate those of the AGA fetuses when maternal blood concentration of glucose is increased acutely (20). No improvement in rates of lipogenesis in the IUGR fetus was found when maternal oxygen concentration was increased. This lack of effect of maternal oxygen supplementation on lipogenesis is consistent with studies by Villee and coworkers $(21,22)$. They demonstrated that the effect of total oxygen deprivation on fetal lipogenesis varied with gestational age. Rates of lipogenesis in liver slices obtained from preterm (15-19 days gestation) rat fetuses incubated anaerobically were reduced to $10 \%$ or less as compared to preterm tissues incubated under aerobic conditions. Similar results were obtained when human fetal tissues were studied at midgestation. However, when tissues were studied at term from either the rat or goat, lipogenesis was not impaired by oxygen deprivation.

The present study suggests that maternal inhalation of supplemental oxygen results in improved growth and survival of the growth-retarded fetus. Since these data demonstrate no improvement in lipogenesis in the IUGR term fetus, it appears that the decreased fetal fatty acid synthesis is related to nutrient (20) rather than oxygen deprivation in pregnancies with impaired uteroplacental blood flow. Although growth and survival in the IUGR fetus can be improved with supplemental maternal oxygen inhalation, lipogenesis does not appear to contribute to either of these improvements.

Acknowledgments. The author is indebted to Ms. G. Clark for skillful technical assistance, Ms. F. Moses and N. Cone for secretarial support, and Drs. J. E. Brazy and A. J. D'Ercole for critical review of this manuscript.

\section{REFERENCES}

1. Baum D, Porte D 1972 A mechanism for regulation of insulin release in hypoxia. Am J Physiol 222:695-699

2. Bligh EG, Dyer WJ 1959 A rapid method of total lipid extraction and 
purification. Can J Biochem Physiol 37:911-917

3. Creasy RK, Barrett CT, De Swiet M 1972 Experimental intrauterine growth retardation in the sheep. Am J Obstet Gynecol 112:566-573

4. Duncan SLB, Lewis BV 1969 Maternal placental and myometrial blood flow in the pregnant rabbit. J Physiol 202:471-481

5. Garvey DJ, Longo LD 1978 Chronic low level maternal carbon monoxide exposure and fetal growth and development. Bio Reprod 19:8-14

6. Gilbert RD, Cummings LA, Juchau MR, et al 1979 Placental diffusing capacity and fetal development in exercising or hypoxic guinea pigs. J Appl Physiol 46:828-834

7. Greiss FC, Anderson SG, King LC 1974 Uterine vascular bed: effects of acute hyperoxia. Am J Obstet Gynecol 118:542-547

8. Longo LD, Yuen P, Gussecki DJ 1973 An aerobic, glycogen-dependent transport of amino acids by the placenta. Nature 243:531-533

9. Lumley JM, Wood C 1967 Influence of hypoxia on glucose transport across the human placenta. Nature 216:403-404

10. McCutcheon IE, Metcalfe J, Metzenberg AB, et al 1982 Organ growth in hyperoxic and hypoxic chick embryos. Respir Physiol 50:153-163

11. Meschia G 1978 Substrate availability in fetal growth. In Naftolin F (ed) Abnormal Fetal Growth: Biological Bases and Consequences. Dahlem Konferenzen, Berlin, pp 221-227

12. Moll W 1973 Placental function and oxygenation in the fetus. Adv Exp Med Biol 37:1017-1026

13. Nitzan M, Orloff S, Schulman JD 1979 Placental transfer of analogs of glucose and amino acids in experimental intrauterine growth retardation. Pediatr
Res 13:100-103

14. O'Shaughnessy RW 1981 Uterine blood flow and fetal growth. In: Van Assche FA, Robertson WB (eds) Fetal Growth Retardation. Churchill-Livingstone, Edinburgh, pp 101-116

15. Power GG, Jenkins $F 1975$ Factors affecting $\mathrm{O}_{2}$ transfer in sheep and rabbit placenta perfused in situ. Am J Physiol 229:1147-1153

16. Robinson JS, Mrcog MB 1979 Growth of the fetus. Br Med Bull 35:137-144

17. Saintonge J, Ross P 1981 Placental blood flow and transfer of nutrient analogs in large, average, and small guinea pig littermates. Pediatr Res 15:152-156

18. Van Geijn HP, Kaylor WM, Nicola KR, et al 1980 Induction of severe intrauterine growth retardation in the Sprague-Dawley rat. Am J Obstet Gynecol 137:43-47

19. Vileisis RA, Fain J, Oh W 1982 Fatty acid synthesis in rat fetuses with intrauterine growth retardation. Metabolism 31:217-222

20. Vileisis RA, Oh W 1983 Effect of increased substrate availability on fatty acid synthesis in the growth retarded fetus. Metabolism 32:90-94

21. Villee CA, Hagerman DD 1958 Effect of oxygen deprivation on metabolism of fetal and adult tissues. Am J Physiol 194:457-464

22. Villee CA, Hagerman DD, Holmberg N, et al 1958 The effects of anoxia on the metabolism of human fetal tissues. Pediatrics 37:953-970

23. Wigglesworth JS 1964 Experimental growth retardation in the foetal rat. J Pathol Bacteriol 88:1-13

24. Wilkening RB, Meschia G 1983 Fetal oxygen uptake, oxygenation, and acidbase balance as a function of uterine blood flow. Am J Physiol 244:H749H755 